\title{
Haag-Ruelle-Hepp Scattering Formalism for Essentially Local Non-Localizable Fields
}

\author{
J. Bümmerstede and W. Lücke \\ Institut für Theoretische Physik der Technischen Universität Clausthal, \\ Clausthal-Zellerfeld, Federal Republic of Germany
}

Received December 8, 1973

\begin{abstract}
We generalize the axiom of locality to the non-localizable case by an axiom of essential locality which means a natural local continuity property of the field commutator on the light-cone. For localizable fields essential locality coincides with ordinary locality while localizable local fields restricted to non-localizable test spaces still remain essentially local. Essential locality is proved to be sufficient for a derivation of the usual Haag-RuelleHepp scattering formalism. Functions of the free field are examples of essentially local fields which may not be localizable.
\end{abstract}

\section{Introduction}

The principal aim of the present paper is to give a generalization of the Haag-Ruelle-Hepp scattering formalism to the non-localizable case, i.e. to the case of test spaces not containing functions with compact support.

In the localizable case causality, usually introduced by local commutativity (in short: locality), is well known to be crucial for the derivation of the asymptotic conditions. Since the standard formulation of locality [20] does not apply to the non-localizable case, we have to look for some other suitable additional assumption.

Thus Steinmann demands the existence of generalized retarded products as defined in [18] and imposes certain regularity properties [19]. Steinmann himself quoted this a purely technical assumption which cannot be given any direct physical interpretation. Moreover, despite extensive research in this field, there does not exist any rigorous proof for the general existence of those Green's functions even in the tempered case (apart from the unrealistic case of two space-time dimensions [17]). It should be interesting, however, to investigate the possibilities of circumventing this difficulty by modification of the defining postulates, extracted from sharp Green's functions for localizable fields, which Steinmann introduced for convenience (compare [18] for this point).

Nevertheless we think it is desirable to work with a more direct substitute of locality which is applicable to both the localizable and the 
non-localizable case. One possibility has been explored by Taylor [22] (see also [23]). According to Taylor a field fulfils the condition of generalised local commutativity if it is a certain limit of local fields. In the present paper we present a (presumably wider) generalization of locality which seems to us more closely related to the usual formulation of micro-causality, at least from a mathematical point of view. This generalized condition will be called essential locality and will, indeed, prove sufficient for a derivation of the usual scattering formalism.

In order to avoid purely technical complications we decide to consider only a single scalar hermitian field $A(x)$ and choose the well known Gelfand spaces $S^{\beta_{1}, \ldots, \beta_{n}}$ to be allowed test spaces [4]. Generalizations will be more or less obvious.

Although our main emphasis is on the non-localizable case, we think the following derivation to be of at least technical interest also for the localizable case.

\section{Locally Continuous Generalized Functions}

As already mentioned in the introduction, we are going to deal with generalized functions on some Gelfand space $S^{\beta_{1}, \cdots, \beta_{n}}$ (see [4] or [15] for the definition and properties of these spaces). Since we are only interested in the completely symmetric case $\beta_{1}=\beta_{2}=\cdots=\beta_{n}$, let us introduce the notation

$$
S^{s}\left(R^{n}\right) \equiv S^{\beta_{1}, \cdots, \beta_{n}}, \quad \beta_{1}=\cdots=\beta_{n}=s
$$

for $s \geqq 0$. We should like to formulate properties of generalized functions on $S^{s}\left(R^{n}\right)$ which, when applied to the localizable case $s>1$, characterize the support of the functional in question. To this end let us introduce two useful definitions ${ }^{1}$ :

Definition. Let $M$ be a closed subset of $R^{n}$ and $S$ a subset of $S^{s}\left(R^{n}\right)$. Then $S$ is called locally bounded on $M$ in $S^{s}\left(R^{n}\right)$ iff there are positive

${ }^{1}$ We use standard notation:

$$
\|\chi\|=\left(\sum_{j=1}^{n}\left(\chi^{j}\right)^{2}\right)^{1 / 2}, \quad \chi=\left(\chi^{1}, \ldots, \chi^{n}\right)
$$

$Z_{+}^{n}=\left\{\alpha=\left(\alpha^{1}, \ldots, \alpha^{n}\right): \alpha^{j}\right.$ non-negative integer $\}$

$$
\begin{gathered}
|\alpha|=\sum_{j=1}^{n} \alpha^{j} ; \quad \chi^{\gamma}=\prod_{j=1}^{n}\left(\chi^{j}\right)^{\nu^{j}} \\
\varphi^{(\alpha)}(\chi)=\left(\frac{\partial}{\partial \chi^{1}}\right)^{\alpha^{1}} \ldots\left(\frac{\partial}{\partial \chi^{n}}\right)^{\alpha^{n}} \varphi\left(\chi^{1}, \ldots, \chi^{n}\right)
\end{gathered}
$$

$U_{e}(M)=\left\{\chi \in R^{n}:\left\|\chi-\chi^{\prime}\right\|<e\right.$ for at least one $\left.\chi^{\prime} \in M\right\}$. 
constants $e$ and $A$ such that for every non-negative integer $N$

$$
\sup _{\varphi \in \mathbb{S}} \sup _{\chi \in U_{e}(M)} \sup _{\alpha \in Z_{+}^{n}} A^{-|\alpha|} \alpha^{-s \alpha}\|\chi\|^{N}\left|\varphi^{(\alpha)}(\chi)\right|<\infty .
$$

Definition. Let $M$ be a closed subset of $R^{n}$ and $F$ a generalized function on $S^{s}\left(R^{n}\right)$. Then, for non-negative $s_{0} \leqq s, F$ is called locally continuous on $M$ with respect to $S^{s_{0}}\left(R^{n}\right)$ iff $\sup _{\varphi \in S}|F(\varphi)|$ is finite for every $S \subset S^{S_{0}}\left(R^{n}\right)$ that is locally bounded on $M$ in $S^{S_{0}}\left(R^{n}\right)$.

Thus suitable properties of the type we are looking for are those of local continuity:

Lemma 1. Let $M$ be a closed subset of $R^{n}$ and let $F$ be a generalized function on $S^{s}\left(R^{n}\right)$. We impose the additional condition $s>1$ and therefore can define $T \equiv \operatorname{supp} F$. Then the following two statements hold:

1. For every non-negative $s_{0} \leqq S$ the restriction of $F$ to $S^{s_{0}}\left(R^{n}\right)$ is locally continuous on $T$ with respect to $S^{s_{0}}\left(R^{n}\right)$.

2. $M \supset T$ if $F$ is locally continuous on $M$ with respect to $S^{s}\left(R^{n}\right)$.

Proof. Since local boundedness on $T$ in $S^{\text {so }}\left(R^{n}\right)$ implies local boundedness on $T$ in $S^{s}\left(R^{n}\right)$, it is sufficient to check the special case $s_{0}=s$. Then the first statement is immediately verified by realizing that, due to strict localizability $(s>1)$, for arbitrary $e_{2}>e_{1}>0$ there are multipliers $k$ on $S^{s}\left(R^{n}\right)$ such that $k(\chi)=1$ for $\chi \in U_{e_{1}}(T)$ and $k(\chi)=0$ for $\chi \notin U_{e_{2}}(T)$, which may be easily constructed by standard techniques (see [3], Appendix 1, for instance). The second statement follows from the fact that $\left\{r \varphi: r \in Z_{+}\right\}$is locally bounded on $M$ in $S^{s}\left(R^{n}\right)$ for every $\varphi \in S^{s}\left(R^{n}\right)$ with $\operatorname{supp} \varphi \subset R^{n}-U_{e}(M), 0<e$ suitable; thus $F(\varphi)=0$.

We need one final result for deriving the cluster property in Section 4:

Lemma 2. Let $M$ be a closed subset of $R^{n}$ and let $F_{0}$ be a multi-linear functional on $S^{s}\left(R^{n}\right) \times S^{s}\left(R^{n_{1}}\right) \times \cdots \times S^{s}\left(R^{n_{l}}\right)$ which is continuous in each variable separately and even locally continuous on $M$ with respect to $S^{s}\left(R^{n}\right)$ in the first argument. Then there is a generalized function $F$ on $S^{s}\left(R^{n+n_{1}+\cdots+n_{l}}\right)$, coinciding with $F_{0}$ on $S^{s}\left(R^{n}\right) \times \cdots \times S^{s}\left(R^{n_{l}}\right)$, which is locally continuous on $M \times R^{n_{1}} \times \cdots \times R^{n_{l}}$ with respect to $S^{S}\left(R^{n+n_{1}+\cdots+n_{l}}\right)$. The linear hull of $S^{s}\left(R^{n}\right) \times \cdots \times S^{s}\left(R^{n_{l}}\right)$ is dense in $S^{s}\left(R^{n+n_{1}+\cdots+n_{l}}\right)$, therefore $F$ is unique.

Proof. See appendix.

\section{Axioms for Essentially Local Fields}

For simplicity we decided to restrict ourselves to one kind of neutral scalar particle with mass $m>0$, described by the hermitian Wightman field $A(x)$ on $S^{s}\left(R^{4}\right), s \geqq 0$. 
This means, the formal integrals

$$
A(\varphi) \equiv \int d x A(x) \varphi(x) ; \quad \varphi \in S^{s}\left(R^{4}\right)
$$

are well defined as (unbounded) operators on a common dense domain $D$ in a separable Hilbert space $\mathscr{H}$, and for $\Phi, \Psi \in D$

$$
\varphi \rightarrow \int d x\langle\Phi|A(x)| \Psi\rangle \varphi(x) \equiv\langle\Phi|A(\varphi)| \Psi\rangle
$$

is a continuous linear functional (generalized function) on $S^{S}\left(R^{4}\right)$. As usual, hermiticity of the field $A(x)$ is defined by

$$
A(\bar{\varphi}) \subset A(\varphi)^{*} \text { for } \varphi \in S^{s}\left(R^{4}\right),
$$

where $\bar{\varphi}$ is the ordinary complex conjugate of $\varphi$. Finally, the following axioms are postulated ${ }^{2}$ :

1. The metric in $\mathscr{H}$ is positive definite.

2. The theory is Poincare covariant, i.e. there is a (strongly) continuous unitary representation $U(\Lambda, a)$ of the connected Poincaré group defined in $\mathscr{H}$, for which we have

$$
U(\Lambda, a) A(x) U(\Lambda, a)^{-1}=A(\Lambda x+a)
$$

in the usual distribution theoretic notation.

3 . There is a vacuum state vector $\Omega$ in $\mathscr{H}$, unique up to a phase factor, such that $P \Omega=0$. Here $P$ is the energy-momentum operator, defined by

$$
U(1, a)=\exp \left(i P_{l} a^{l}\right) .
$$

4. $A(\varphi) D \subset D$ for $\varphi \in S^{s}\left(R^{4}\right)$. Therefore, according to Lemma 2, the expectation values $\left\langle\Phi\left|A\left(x_{1}\right) \ldots A\left(x_{n}\right)\right| \Psi\right\rangle(\Phi, \Psi \in D)$ and especially the vacuum expectation values (VEV)

$$
\mathfrak{W}\left(x_{1}, \ldots, x_{n}\right) \equiv\left\langle\Omega\left|A\left(x_{1}\right) \ldots A\left(x_{n}\right)\right| \Omega\right\rangle
$$

are uniquely defined as generalized functions on $S^{\mathrm{s}}\left(R^{4 n}\right)$.

5. The field is essentially local, i.e. for $\Phi, \Psi \in D$ the generalized function $\left\langle\Phi\left|[A(x), A(y)]_{-}\right| \Psi\right\rangle$ is locally continuous on ${ }^{3}\left\{(x, y) \in R^{8}: x\right.$ $-y \in \bar{V}\}$ with respect to $S^{\mathrm{s}}\left(R^{8}\right)$.

6. Apart from the eigenvalue 0 corresponding to the vacuum, the spectrum of $P$ is contained in $\overline{V_{+}^{m}}$.

By Lemma 1 we see Axiom 5 to be equivalent to Einstein causality [8] in the localizable case, i.e.

$$
\operatorname{supp}\left\langle\Phi\left|[A(x), A(y)]_{-}\right| \Psi\right\rangle \subset\left\{(x, y) \in R^{8}: x-y \in \bar{V}\right\}
$$

\footnotetext{
2 We closely follow Ruelle's formulation [21].

${ }^{3}$ As usual, we denote by $\bar{V}$ the closed light-cone and by $\overline{V_{ \pm}^{m}}$ the set $\left\{p \in R^{4}: \pm p^{0}>0\right.$, $\left.p^{2} \geqq m^{2}\right\}$.
} 
for $s>1$. On the other hand ${ }^{4}$, again by Lemma 1, a local field $A(x)$ on $S^{s}\left(R^{4}\right)(s>1)$ still remains essentially local when restricted to $S^{s_{0}}\left(R^{4}\right)$, $s_{0}<s$. Therefore we feel justified to postulate essential locality as a substitute for locality, which cannot be formulated any more in the non-localizable case $s<1$. We expect that many results for local fields can still be proved for essentially local non-localizable fields.

In the present paper we will check the Haag-Ruelle-Hepp theory from this point of view and therefore need one further assumption concerning the particle interpretation of the theory. As in [13] we assume the following structure of the 2-point function:

$$
\langle\Omega|A(x) A(y)| \Omega\rangle=i \Delta_{m}^{+}(x-y)+i \int_{M}^{\infty} d \varrho(\mu) \Delta_{\mu}^{+}(x-y), \quad M>m .
$$

\section{Cluster Property}

For essentially local fields, apart from some minor alterations, we still can use Ruelle's method [21] to prove the well-known cluster properties first conjectured by Haag [6]. Here again, we want to keep things as simple as possible. Therefore we will not prove the cluster property in its most general form but just prove it in the form which is sufficient for the derivation of Haag's asymptotic condition ${ }^{5}$.

First of all we have to exploit essential locality for the truncated vacuum expectation values (TVEV):

Lemma 3. Let $n, n^{\prime}$ be positive integers with $n^{\prime}<n$. Then the generalized function $\left\langle\Omega\left|A\left(x_{1}\right) \ldots\left[A\left(x_{n^{\prime}}\right), A\left(x_{n^{\prime}+1}\right)\right]_{-} \ldots\right| \Omega\right\rangle^{T}$ is locally continuous on ${ }^{6}$ $\left\{\hat{x} \in R^{4 n}: x_{n^{\prime}}-x_{n^{\prime}+1} \in \bar{V}\right\}$ with respect to $S^{s}\left(R^{4 n}\right)$.

Proof. Remember that the TVEV

$$
\mathfrak{B}^{T}(\hat{x}) \equiv\left\langle\Omega\left|A\left(x_{1}\right) \ldots A\left(x_{n}\right)\right| \Omega\right\rangle^{T},
$$

while recursively defined by

$$
\mathfrak{B}(\hat{x})=\sum_{l=1}^{n} \sum_{M \in P_{l}(n)} \prod_{J \in M} \mathfrak{B}^{T}\left(x_{j_{1}}, \ldots, x_{j_{k_{J}}}\right),
$$

are explicitely given by the $\mathrm{VEV}$ via

$$
\mathfrak{W}^{T}(\hat{x})=\sum_{l=1}^{n}(-1)^{l-1}(l-1) ! \sum_{M \in P_{l}(n)} \prod_{J \in M} \mathfrak{M}\left(x_{j_{1}}, \ldots, x_{j_{k_{J}}}\right) .
$$

${ }^{4}$ It should be of some interest to find out the necessary and sufficient conditions for a field on $S^{s}\left(R^{4}\right)$ in case $s>1$ to be essentially local when restricted to $S^{s_{0}}\left(R^{4}\right)$, where $s_{0}<1$. However, we will not go into this problem, here.

5 There do not arise new difficulties in the proof of the general form, but it is only the notation that is more involved [21].

${ }^{6}$ We write $\hat{x}=\left(x_{1}, \ldots, x_{n}\right)$ with $x_{r}=\left(x_{r}^{0}, x_{r}\right)=\left(x_{r}^{0}, x_{r}^{1}, x_{r}^{2}, x_{r}^{3}\right) \in R^{4}$. 
Here $M=\left\{J_{1}, \ldots, J_{l}\right\} \in P_{l}(n)$ iff it is a partition of $\{1, \ldots, n\}$ into $l$ (nonempty) disjoint ordered subsets $J_{r}$ with the ordering in each $J=\left(j_{1}, \ldots, j_{k_{J}}\right)$ being the natural relative ordering of integers. Now, if in the expression for $\mathfrak{B}^{T}$ we take the sum only over such $M$ for which $x_{n^{\prime}}$ and $x_{n^{\prime}+1}$ belong to different $J \in M$, we obtain a generalized function which is obviously symmetric in the arguments $x_{n^{\prime}}$ and $x_{n^{\prime}+1}$. This part cannot contribute to

$$
\left\langle\Omega\left|A\left(x_{1}\right) \ldots\left[A\left(x_{n^{\prime}}\right), A\left(x_{n^{\prime}+1}\right)\right]_{-} \ldots A\left(x_{n}\right)\right| \Omega\right\rangle^{T} .
$$

Thus, according to Axioms 4 and 5, the statement of Lemma 3 follows by Lemma 2.

For the sequel it is convenient to introduce some short-hand notation: If $\pi$ is a permutation of $(1, \ldots, n)$ then we write

$$
\mathfrak{W}_{\pi}^{T}(\hat{x}) \equiv \mathfrak{W}^{T}\left(x_{\pi(1)}, \ldots, x_{\pi(n)}\right) .
$$

If, moreover, $\varphi \in S^{s}\left(R^{4 n}\right)$ and $a_{1}, \ldots, a_{n} \in R^{3}$ we write

$$
\mathfrak{F}_{\varphi}^{\pi}\left(\hat{a}_{0}\right) \equiv \int d \hat{x} \mathfrak{W}_{\pi}^{T}(\hat{x}) \varphi\left(\hat{x}-\hat{a}_{0}\right)
$$

with $d \hat{x} \equiv d x_{1} \ldots d x_{n}, \hat{a}_{0} \equiv\left(\left(0, \boldsymbol{a}_{1}\right), \ldots,\left(0, \boldsymbol{a}_{n}\right)\right)$. For positive $n, n^{\prime} \in Z_{+}\left(\equiv Z_{+}^{1}\right)$ with $n^{\prime}<n$ we denote by $S_{n, n^{\prime}}$ the set of all permutations $\pi$ of $(1, \ldots, n)$ fulfilling the inequality $\pi(r)<\pi\left(r^{\prime}\right)$ for $0<r<r^{\prime} \leqq n^{\prime}$ as well as for $n^{\prime}<r<r^{\prime} \leqq n$. Finally, for $\pi \in S_{n, n^{\prime}}$ and $e>0$ we define

$$
\begin{aligned}
K_{e}^{\pi} \equiv & \left\{\hat{x} \in R^{4 n}: x_{\pi(r)}-x_{\pi\left(r^{\prime}\right)} \in U_{e}(\bar{V})\right. \text { for } \\
& \text { suitable } \left.\quad r, r^{\prime} \in Z_{+} \text {with } 0<r \leqq n^{\prime}<r^{\prime} \leqq n\right\}
\end{aligned}
$$

and

$$
m_{\pi, \hat{a}_{0}} \equiv \min _{r \in\left\{1, \ldots, n^{\prime}\right\}} \min _{r^{\prime} \in\left\{n^{\prime}+1, \ldots, n\right\}}\left\|\boldsymbol{a}_{\pi(r)}-\boldsymbol{a}_{\pi\left(r^{\prime}\right)}\right\| .
$$

Now we are prepared for the first basic step in Ruelle's proof:

Lemma 4. Let $N$ be a non-negative integer and let $\pi \in S_{n, n^{\prime}}$ and $\varphi \in S^{S}\left(R^{4 n}\right)$. Then there is a constant $C$ for which the inequality

$$
m_{\pi, \hat{a}_{0}}^{N}\left|\mathfrak{F}_{\varphi}^{1}\left(\hat{a}_{0}\right)-\mathfrak{F}_{\varphi}^{\pi}\left(\hat{a}_{0}\right)\right|<C
$$

holds for arbitrary $\boldsymbol{a}_{1}, \ldots, \boldsymbol{a}_{n} \in R^{3}$ with $\left\|\boldsymbol{a}_{r}-\boldsymbol{a}_{\boldsymbol{r}^{\prime}}\right\| \leqq(n-1) m_{\pi, \hat{a}_{0}}$.

Proof. Since $\pi \in S_{n, n^{\prime}}, \mathfrak{W}^{T}(\hat{x})-\mathfrak{W}_{\pi}^{T}(\hat{x})$ can be written as a finite sum of generalized functions of the form

$$
\left\langle\Omega\left|A\left(x_{\pi^{\prime}(1)}\right) \ldots\left[A\left(x_{\pi^{\prime}(l)}\right), A\left(x_{\pi^{\prime}(l+1)}\right)\right]_{-} \ldots A\left(x_{\pi^{\prime}(n)}\right)\right| \Omega\right\rangle^{T}
$$

with suitable $l \in\{1, \ldots, n-1\}$ and suitable permutations $\pi^{\prime}$ of $(1, \ldots, n)$ for which $\pi^{\prime}(l) \in\left\{\pi\left(n^{\prime}+1\right), \ldots, \pi(n)\right\}$ and $\pi^{\prime}(l+1) \in\left\{\pi(1), \ldots, \pi\left(n^{\prime}\right)\right\}$. Hence, by Lemma $3, \mathfrak{W}^{T}(\hat{x})-\mathfrak{B}_{\pi}^{T}(\hat{x})$ is locally continuous on $K_{e}^{\pi}$ (for arbitrary 
$e>0$ ) with respect to $S^{s}\left(R^{4 n}\right)$. Therefore, by translation invariance of the theory, it is sufficient to prove that

$$
\left\{\varphi_{\hat{a}_{0}}(\hat{x}) \equiv m_{\pi, \hat{a}_{0}}^{N} \varphi\left(\hat{x}-\hat{a}_{0}\right): \boldsymbol{a}_{2}, \ldots, \boldsymbol{a}_{n} \in R^{3}, \boldsymbol{a}_{1} \equiv 0\right\}
$$

is locally bounded on $K_{e}^{\pi}(e>0)$ in $S^{s}\left(R^{4 n}\right)$. This, however is an easy consequence of the following estimate:

$$
\begin{aligned}
& \sup _{\hat{x} \in K_{e}^{\tau}}\|\hat{x}\|^{N^{\prime}}\left|\varphi_{\hat{a}_{0}}^{(\hat{\alpha})}(\hat{x})\right| \\
& \leqq m_{\pi, \hat{a}_{0}}^{N} \sup _{\hat{x}+\hat{a}_{0} \in K_{e}^{\pi}}\left\|\hat{x}+\hat{a}_{0}\right\|^{N^{\prime}}\left|\varphi^{(\hat{\alpha})}(\hat{x})\right| \\
& \leqq m_{\pi, \hat{a}_{0}}^{N} \sup _{\hat{x}+\hat{a}_{0} \in K_{e}^{\pi}}\left(\|\hat{x}\|+(n-1)^{2} m_{\pi, \hat{a}_{0}}\right)^{N^{\prime}}\left|\varphi^{(\hat{\alpha})}(\hat{x})\right| \\
& \leqq\left(1+4(n-1)^{2}\right)^{N+N^{\prime}} \sup _{\hat{x} \in R^{4 n}}(\|\hat{x}\|+e)^{N+N^{\prime}}\left|\varphi^{(\hat{\alpha})}(\hat{x})\right| .
\end{aligned}
$$

In the latter inequality we used that $m_{\pi, \hat{a}_{0}} \leqq 4(\|\hat{x}\|+e)$ if $\hat{x}+\hat{a}_{0} \in K_{e}^{\pi}$.

Theorem 1 (cluster property). Let $N$ be a non-negative integer and let $\varphi$ be a test function from $S^{s}\left(R^{4 n}\right)$. Then there is a constant $C$ such that the inequality

$$
\left\|\hat{a}_{0}\right\|^{N}\left|\mathfrak{F}_{\varphi}^{1}\left(\hat{a}_{0}\right)\right|<C
$$

holds for $\boldsymbol{a}_{1}=0$ and arbitrary $\boldsymbol{a}_{2}, \ldots, \boldsymbol{a}_{n} \in R^{3}$.

Remark. This theorem shows that $\mathfrak{F}_{\varphi}^{1}\left(\hat{a}_{0}\right)$ is a tempered function in the difference variables $a_{1}-a_{2}, \ldots, a_{n-1}-a_{n}\left(a_{1} \in R^{3}\right)$.

Proof. Since for every configuration $a_{2}, \ldots, a_{n}$ there is a positive integer $n^{\prime}<n$ and a permutation $\pi \in S_{n, n^{\prime}}$ such that $\left\|\hat{a}_{r}-\hat{a}_{r^{\prime}}\right\| \leqq(n-1) m_{\pi, \hat{a}_{0}}$, by Lemma 4 it is quite sufficient to prove, for arbitrary but fixed $\pi \in S_{n, n^{\prime}}$, that

$$
m_{\pi, \hat{a}_{0}}^{N}\left|\mathfrak{F}_{\varphi}^{\pi}\left(\hat{a}_{0}\right)\right|
$$

is uniformly bounded in $\boldsymbol{a}_{2}, \ldots, \boldsymbol{a}_{n} \in R^{3}$, provided $\left\|\boldsymbol{a}_{r}-\boldsymbol{a}_{r^{\prime}}\right\| \leqq(n-1) m_{\pi, \hat{a}_{0}}$ holds for $r, r^{\prime} \in\{1, \ldots, n\}$. In order to prove this, let us introduce another permutation $\pi^{\prime}$ by

$$
\left(\pi^{\prime}(1), \ldots, \pi^{\prime}(n)\right)=\left(\pi\left(n^{\prime}+1\right), \ldots, \pi(n), \pi(1), \ldots, \pi\left(n^{\prime}\right)\right) .
$$

Then, by Lemma 4,

$$
m_{\pi, \hat{a}_{0}}^{N}\left|\mathfrak{F}_{\psi}^{\pi}\left(\hat{a}_{0}\right)-\mathfrak{F}_{\psi}^{\pi \prime}\left(\hat{a}_{0}\right)\right|
$$

is uniformly bounded in $a_{2}, \ldots, a_{n} \in R^{3}$ (remember $a_{1}=0$ ) for fixed $\psi \in S^{s}\left(R^{4 n}\right)$, provided $\left\|\boldsymbol{a}_{r}-\boldsymbol{a}_{r^{\prime}}\right\| \leqq(n-1) m_{\pi, \hat{a}_{0}}$.

Therefore, the theorem is proved if we find a test function $\psi \in S^{S}\left(R^{4 n}\right)$ for which $\mathfrak{F}_{\psi}^{\pi}\left(\hat{a}_{0}\right)=\mathfrak{F}_{\varphi}^{\pi}\left(\hat{a}_{0}\right)$ and $\mathfrak{F}_{\psi}^{\pi^{\prime}}\left(\hat{a}_{0}\right)=0$ : 
Exactly as for tempered fields (see [12], Chapter III, $\S \S 3 \mathrm{E}$ and 5C) it can be proved that

$$
\operatorname{supp} \widetilde{\mathfrak{W}^{T}} \subset\left\{\hat{p} \in R^{4 n}: p_{1}+\cdots+p_{n}=0, \sum_{r=1}^{k} p_{r} \in \overline{V_{+}^{m}} \text { for } k<n\right\},
$$

where the Fourier transform $\overline{\mathfrak{M}^{T}}$ of $\mathfrak{B}^{T}$ is formally defined by ${ }^{7}$

Consequently:

$$
\widetilde{\mathfrak{W}^{T}}(\hat{p})=(2 \pi)^{-2 n} \int d \hat{x} \exp \left(i \sum_{r=1}^{n} p_{r} x_{r}\right) \mathfrak{W}^{T}(\hat{x})
$$

$$
\begin{aligned}
& \operatorname{supp} \widetilde{\mathfrak{B}_{\pi}^{T}} \subset\left\{\hat{p} \in R^{4 n}: \sum_{r=1}^{n^{\prime}} p_{\pi(r)} \in \overline{V_{+}^{m}}\right\} \\
& \operatorname{supp} \widetilde{\mathfrak{M}_{\pi^{\prime}}^{T}} \subset\left\{\hat{p} \in R^{4 n}: \sum_{r=1}^{n^{\prime}} p_{\pi(r)} \in \overline{V_{-}^{m}}\right\} .
\end{aligned}
$$

Therefore, a suitable choice for $\psi$ is given by

$$
\tilde{\psi}(\hat{p}) \equiv \tilde{h}\left(\sum_{r=1}^{n^{\prime}} p_{\pi(r)}\right) \tilde{\varphi}(\hat{p}),
$$

where $\tilde{h} \in \mathcal{O}_{\mathrm{M}}\left(R^{4}\right)$ is equal to 1 on $\overline{V_{-}^{m}}$ and vanishes on $\overline{V_{+}^{m}}$.

\section{Asymptotic Conditions}

The derivation of the well-known asymptotic conditions can be traced back to the methods used for tempered fields [13], by means of the following

Lemma 5. Let $n, n^{\prime}$ be positive integers with $n^{\prime} \leqq n$ and let $S$ be a finite subset of $S^{s}\left(R^{n}\right)$. Then there is a function $g \in S^{s}\left(R^{n^{\prime}}\right)$ such that for every $\varphi \in S$

$$
\tilde{h}_{\varphi}(\chi) \equiv \tilde{\varphi}\left(\chi^{1}, \ldots, \chi^{n}\right) / \tilde{g}\left(\chi^{1}, \ldots, \chi^{n^{\prime}}\right), \chi \in R^{n}
$$

defines a test function in $\widetilde{S^{s}}\left(R^{n}\right)$.

Proof. Since $\widetilde{S^{0}}\left(R^{n}\right)=\mathscr{D}\left(R^{n}\right)$, the statement is trivial for $s=0$. Now suppose $s>0$. Then $\varphi \in S^{s}\left(R^{n}\right)$ iff [4]:

$$
\tilde{\varphi}(\chi) \exp \left(+a\left(1+\|\chi\|^{2}\right)^{\frac{1}{2 s}}\right) \in \mathscr{S}\left(R^{n}\right) \text { for suitable } a>0 \text {. }
$$

Since $S$ is finite, $a$ may be chosen once for all $\varphi \in S$. Therefore, with

$$
\tilde{g}\left(\chi^{1}, \ldots, \chi^{n^{\prime}}\right) \equiv \exp \left(-\frac{a}{2}\left(1+\left\|\left(\chi^{1}, \ldots, \chi^{n^{\prime}}\right)\right\|^{2}\right)^{\frac{1}{2 s}}\right) \in \widetilde{S^{s}}\left(R^{n^{\prime}}\right),
$$

\footnotetext{
7 Note the different sign in the exponential as compared to [12].
} 
we have

$$
\tilde{h}_{\varphi}(\chi) \equiv \tilde{\varphi}(\chi) / \tilde{g}\left(\chi^{1}, \ldots, \chi^{n^{\prime}}\right) \in \widetilde{S}^{s}\left(R^{n}\right) \quad \text { for } \quad \varphi \in S .
$$

Although each of the various steps in the following derivation was already explicitely carried out in at least one of the Ref. [1, 6, 10, 13 or 21], we think it worthwhile fitting them all together. We will use the same notation as in [13], i.e. for $\varphi \in S^{s}\left(R^{4}\right)$ :

$$
\begin{gathered}
\tilde{\varphi}(p) \equiv(2 \pi)^{-2} \int d x \varphi(x) e^{i p x} \\
\omega_{\boldsymbol{p}} \equiv\left(\boldsymbol{p}^{2}+m^{2}\right)^{1 / 2} \\
\varphi(x ; t) \equiv(2 \pi)^{-5 / 2} \int d p \tilde{\varphi}(p)\left(\frac{p^{0}+\omega_{\boldsymbol{p}}}{2 \omega_{\boldsymbol{p}}}\right) e^{i\left(p^{0}-\omega_{\boldsymbol{p}}\right) t} e^{-i p x} \\
A(\varphi ; t) \equiv \int d x A(x) \varphi^{*}(x ; t) .
\end{gathered}
$$

We will also refer to the following

Definition. A set $\left\{\hat{f_{j}}\right\} \subset \mathscr{S}\left(R^{3}\right)$ is called essentially non-overlapping if $\operatorname{supp} \hat{f}_{j} \cap \operatorname{supp} \hat{f}_{l}$ has no interior points for $j \neq l$.

As a first step we have to realize some physically plausible properties of smooth Klein-Gordon wave functions:

Lemma 6. Let $\hat{f} \in \mathscr{S}\left(R^{3}\right)$. Then there is a constant $C$ such that the Klein-Gordon wave function

$$
f(x) \equiv(2 \pi)^{-3 / 2} \int \frac{d \boldsymbol{p}}{2 \omega_{\boldsymbol{p}}} \hat{f}(\boldsymbol{p}) e^{-i\left(\omega_{\boldsymbol{p}} x^{0}-\boldsymbol{p} \boldsymbol{x}\right)}
$$

fulfils the inequalities

and

$$
\left|x^{0}\right|^{3 / 2}|f(x)|<C
$$

$$
\left(1+\left|x^{0}\right|\right)^{-3 / 2} \int d \boldsymbol{x}|f(x)|<C
$$

for arbitrary $x \in R^{4}$.

Proof. See [1], Lemma 14.1.

Lemma 7. Let $\left\{\hat{f}_{1}, \hat{f}_{2}\right\} \subset \mathscr{S}\left(R^{3}\right)$ be essentially non-overlapping and let $N$ be a non-negative integer. Then the Klein-Gordon wave functions

$$
f_{j}(x) \equiv(2 \pi)^{-3 / 2} \int \frac{d \boldsymbol{p}}{2 \omega_{\boldsymbol{p}}} \hat{f}_{j}(\boldsymbol{p}) e^{-i\left(\omega_{\boldsymbol{p}} x^{0}-\boldsymbol{p} \boldsymbol{x}\right)}
$$

fulfil the inequality

$$
\left|t^{N} f_{1}\left(t, x_{1}\right) f_{2}\left(t, x_{2}\right)\right|<C
$$

for arbitrary $t \in R^{1}$ and $\boldsymbol{x}_{1}, \boldsymbol{x}_{2} \in R^{3}$ with $\left\|\boldsymbol{x}_{1}-\boldsymbol{x}_{2}\right\|^{2}<|t|$. 
Proof. See [13], Lemma 3.

By these lemmas, if we define

$$
\begin{aligned}
& P^{\prime}(n) \equiv \emptyset \quad \text { for } n \text { odd, } \\
& P^{\prime}(n) \equiv\left\{M \in P_{n / 2}(n): k_{J}=2 \text { for all } J \in M\right\} \text { for } n \text { even, }
\end{aligned}
$$

we can derive the following key result:

Theorem 2. Let $\varphi_{1}, \ldots, \varphi_{n}$ be test functions from $S^{s}\left(R^{4}\right)$. Then there is a constant $C$ for which the inequality ${ }^{8}$

$$
\begin{gathered}
\sup _{t \in R^{1}}|t|^{g} \mid\left\langle\Omega\left|\prod_{j=1}^{n} A^{\#}\left(\varphi_{j} ; t\right)\right| \Omega\right\rangle \\
-\sum_{M \in P^{\prime}(n)} \prod_{J \in M}\left\langle\Omega\left|A^{\#}\left(\varphi_{j_{1}} ; 0\right) A^{\#}\left(\varphi_{j_{2}} ; 0\right)\right| \Omega\right\rangle \mid<C
\end{gathered}
$$

holds with

$$
g= \begin{cases}3 / 2 & \text { for } n \text { odd } \\ 3 & \text { for } n \text { even } .\end{cases}
$$

If, moreover, $\tilde{\varphi}_{j}(p)=\hat{f}_{j}(p) \tilde{\psi}(p) \quad(j=1, \ldots, n)$ for some $\psi \in S^{s}\left(R^{4}\right)$ and $\hat{f}_{1}, \ldots, \hat{f}_{n} \in \mathscr{S}\left(R^{3}\right)$ with at least two of any three $\hat{f}_{j_{1}}, \hat{f}_{j_{2}}, \hat{f}_{j_{3}}$ forming an essentially non-overlapping set, then there is a sequence of constants $C_{0}, C_{1}, \ldots$ such that even

$$
\begin{gathered}
\sup _{t \in R^{1}}|t|^{N} \mid\left\langle\Omega\left|\prod_{j=1}^{n} A^{\#}\left(\varphi_{j} ; t\right)\right| \Omega\right\rangle \\
-\sum_{M \in P^{\prime}(n)} \prod_{J \in M}\left\langle\Omega\left|A^{\#}\left(\varphi_{j_{1}} ; 0\right) A^{\#}\left(\varphi_{j_{2}} ; 0\right)\right| \Omega\right\rangle \mid<C_{N}
\end{gathered}
$$

holds for all $N \in Z_{+}$.

Proof. Anyway, by Lemma 5, the $\tilde{\varphi}_{j}$ may be represented in the form $\tilde{\varphi}_{j}(p)=\hat{f}_{j}(\boldsymbol{p}) \tilde{\psi}_{j}(p)$ with $\left\{\psi_{1}, \ldots, \psi_{n}\right\} \subset S^{s}\left(R^{4}\right)$ and $\left\{\hat{f}_{1}, \ldots, \hat{f}_{n}\right\} \subset \mathscr{S}\left(R^{3}\right)$ not necessarily essentially non-overlapping. Now, by use of the almost localized fields

$$
B_{j}(x) \equiv(2 \pi)^{-2} \int d x^{\prime} A\left(x^{\prime}\right) \psi_{j}\left(x^{\prime}-x\right)
$$

and the smooth positive frequency Klein-Gordon wave functions

we may write

$$
f_{j}(x) \equiv(2 \pi)^{-3 / 2} \int \frac{d \boldsymbol{p}}{2 \omega_{\boldsymbol{p}}} \hat{f}_{j}(\boldsymbol{p}) e^{-i\left(\omega_{\boldsymbol{p}} x^{0}-\boldsymbol{p} \boldsymbol{x}\right)}
$$

$$
A^{*}\left(\varphi_{j} ; t\right)=i \int_{x^{0}=t} d \boldsymbol{x}\left(B_{j}(x)-\frac{\partial f_{j}(x)}{\partial x^{0}}-\frac{\partial B_{j}(x)}{\partial x^{0}} f_{j}(x)\right) \text { on } D
$$

\footnotetext{
${ }^{8}$ We write $A^{\#}\left(\varphi_{j} ; t\right)$, meaning either $A\left(\varphi_{j} ; t\right)$ or $A^{*}\left(\varphi_{j} ; t\right)$ throughout.
} 
for $j=1, \ldots, n$. Expanding the first term of

$$
\begin{aligned}
W \equiv & \left\langle\Omega\left|\prod_{j=1}^{n} A^{\#}\left(\varphi_{j} ; t\right)\right| \Omega\right\rangle \\
& -\sum_{M \in P^{\prime}(n)} \prod_{J \in M}\left\langle\Omega\left|A^{\#}\left(\varphi_{j_{1}} ; 0\right) A^{\#}\left(\varphi_{j_{2}} ; 0\right)\right| \Omega\right\rangle
\end{aligned}
$$

into the corresponding sum of products of TVEV (see proof of Lemma 3), we therefore see $W$ to be a finite polynomial in "variables" of the form

$$
I(t)=\int d x_{1} \ldots d x_{k} f_{1}^{\prime}\left(t, x_{1}\right) \ldots f_{k}^{\prime}\left(t, x_{k}\right) \mathfrak{F}_{\varphi^{\prime}}^{1}\left(\left(t, x_{1}\right), \ldots,\left(t, x_{k}\right)\right)
$$

where $f_{r}^{\prime} \in\left\{f_{\pi(r)}, f_{\pi(r)}^{*}, \partial_{0} f_{\pi(r)}, \partial_{0} f_{\pi(r)}^{*}\right\}, r=1, \ldots, k$, for some permutation $\pi$ of $\{1, \ldots, n\}$ and

$$
\varphi^{\prime}\left(x_{1}, \ldots, x_{k}\right)=\prod_{r=1}^{k} \psi_{r}^{\prime}\left(x_{r}\right)
$$

with $\psi_{r}^{\prime} \in\left\{\psi_{l^{\prime}}, \psi_{l^{\prime}}^{*}, \partial_{0} \psi_{l^{\prime}}, \partial_{0} \psi_{l^{\prime}}^{*}\right\}$ for suitable $l^{\prime}=l^{\prime}(r)$.

Products of factors $I(t)$ with at least one $k=1$ or, alternatively, all $k<3$ do not contribute because (3.1) implies

$$
\begin{aligned}
A\left(\varphi_{j} ; t\right) \Omega & =0 \\
\left\langle\Omega\left|A^{\#}\left(\varphi_{j_{1}} ; t\right) A^{\#}\left(\varphi_{j_{2}} ; t\right)\right| \Omega\right\rangle & =\left\langle\Omega\left|A^{\#}\left(\varphi_{j_{1}} ; 0\right) A^{\#}\left(\varphi_{j_{2}} ; 0\right)\right| \Omega\right\rangle .
\end{aligned}
$$

If $n$ is even, in each non-vanishing product we even have either a factor $I(t)$ with $k>3$ or two factors $I(t)$ with $k=3$. Consequently, the theorem is proved if the following statements are verified $(k>1)$ :

(i) $\sup _{t \in R^{1}}|t|^{3(k-2) / 2}|I(t)|<\infty$,

(ii) if $k \geqq 3$ and if at least two of any three $\hat{f}_{j_{1}}, \hat{f}_{j_{2}}, \hat{f}_{j_{3}}$ form an essentially non-overlapping set, then even

$$
\sup _{t \in R^{1}}|t|^{N}|I(t)|<\infty
$$

holds for all $N \in Z_{+}$.

Let us define

$$
\hat{x}_{00} \equiv\left(0,\left(0, x_{2}\right), \ldots,\left(0, x_{k}\right)\right) \in R^{4 k} .
$$

Then, by translation invariance of the theory and by Theorem 1, we have:

$$
\begin{aligned}
|I(t)| & \leqq \int d \boldsymbol{x}_{1} \ldots d \boldsymbol{x}_{k}\left|f_{1}^{\prime}\left(t, \boldsymbol{x}_{1}\right) f_{2}^{\prime}\left(t, \boldsymbol{x}_{1}+\boldsymbol{x}_{2}\right) \ldots f_{k}^{\prime}\left(t, \boldsymbol{x}_{1}+\boldsymbol{x}_{k}\right) \mathfrak{F}_{\varphi^{\prime}}^{1}\left(\hat{x}_{00}\right)\right| \\
& \leqq \int d \boldsymbol{x}_{1}\left|f_{1}^{\prime}\left(t, \boldsymbol{x}_{1}\right)\right| \prod_{r=2}^{k} \max _{\boldsymbol{x}_{r}^{\prime} \in \boldsymbol{R}^{3}}\left|f_{r}^{\prime}\left(t, \boldsymbol{x}_{r}^{\prime}\right)\right| \int d \boldsymbol{x}_{2} \ldots d \boldsymbol{x}_{k}\left|\mathfrak{F}_{\varphi^{\prime}}^{1}\left(\hat{x}_{00}\right)\right| .
\end{aligned}
$$

Hence statement (i) follows from Lemma 6. On the other hand, $\pi(1)$, $\pi(2)$, and $\pi(3)$ are pairwise different. If $\hat{f}_{1}, \ldots, \hat{f}_{n}$ meet the requirements of the second part of the theorem, therefore, by Lemma 7 there are 
$r, r^{\prime} \in\{1,2,3\}$ such that for every $N^{\prime} \in Z_{+}$there is a constant $C$ for which the inequality

$$
\left|t^{N^{\prime}} f_{r}^{\prime}\left(t, x_{r}\right) f_{r^{\prime}}^{\prime}\left(t, x_{r^{\prime}}\right)\right|<C
$$

holds for arbitrary $t \in R^{1}$ and arbitrary $x_{r}, x_{r^{\prime}} \in R^{3}$ with $\left\|x_{r}-x_{r^{\prime}}\right\|^{2}<|t|$. Without loss of generality we may assume $r=2, r^{\prime}=3$. Moreover, by Theorem 1 again, for every $N^{\prime} \in Z_{+}$there is a constant $C^{\prime}$ for which the inequality

$$
\left|t^{N^{\prime}} \mathfrak{F}_{\varphi^{\prime}}^{1}\left(\hat{x}_{00}\right)\right|<C^{\prime}
$$

holds for arbitrary $t \in R^{1}$ and arbitrary $x_{2}, \ldots, x_{k} \in R^{3}$ with $\left\|x_{2}-x_{3}\right\|^{2}$ $\geqq|t|$. Therefore statement (ii) is a consequence of Lemma 6 and the inequality

$$
\begin{aligned}
|I(t)| \leqq & \max _{\substack{\boldsymbol{x}_{2}, \ldots, \boldsymbol{x}_{k} \in R^{3} \\
\left\|\boldsymbol{x}_{2}-\boldsymbol{x}_{3}\right\|^{2} \geqq|t|}}\left|\mathfrak{F}_{\varphi^{\prime}}^{1}\left(\hat{x}_{00}\right)\right| \int d \boldsymbol{x}_{1}^{\prime} \ldots d \boldsymbol{x}_{k}^{\prime}\left|f_{1}^{\prime}\left(t, \boldsymbol{x}_{1}^{\prime}\right) \ldots f_{k}^{\prime}\left(t, \boldsymbol{x}_{k}^{\prime}\right)\right| \\
& +\max _{\substack{\boldsymbol{x}_{2}^{\prime}, \ldots, \boldsymbol{x}_{k} \in R^{3} \\
\left\|\boldsymbol{x}_{2}^{\prime}-\boldsymbol{x}_{3}^{\prime}\right\|^{2}<|t|}} \prod_{r=2}^{k}\left|f_{r}^{\prime}\left(t, \boldsymbol{x}_{r}^{\prime}\right)\right| \int d \boldsymbol{x}_{1}\left|f_{1}^{\prime}\left(t, \boldsymbol{x}_{1}\right)\right| \int d \boldsymbol{x}_{2} \ldots d \boldsymbol{x}_{k}\left|\mathfrak{F}_{\varphi^{\prime}}^{1}\left(\hat{x}_{00}\right)\right| .
\end{aligned}
$$

This completes the proof of Theorem 2.

From Theorem 2 we can conclude the following very special form of

Haag's Strong Asymptotic Condition. Let $\varphi_{1}, \ldots, \varphi_{n}$ be test functions from $S^{s}\left(R^{4}\right)$ with supp $\tilde{\varphi}_{j} \subset\left\{p \in R^{4}: p^{2}<M^{2}\right\}$. Then the strong limit

$$
\mathrm{s}-\lim _{t \rightarrow \pm \infty} \prod_{j=1}^{n} A^{*}\left(\varphi_{j} ; t\right) \Omega
$$

exists in $\mathscr{H}$ and represents an asymptotic state corresponding to $n$ particles with momentum space wave functions $\hat{f}_{j}(\boldsymbol{p}) \equiv \tilde{\varphi}_{j}\left(\omega_{\boldsymbol{p}}, \boldsymbol{p}\right)$.

Proof. By Theorem 2 we see that

$$
\left\|\frac{d}{d t} \prod_{j=1}^{n} A^{*}\left(\varphi_{j} ; t\right) \Omega\right\|^{2},
$$

which is the VEV of an even number of field operators, decreases like $|t|^{-3}$ for $t \rightarrow \pm \infty$. This is because the subtraction of products of 2-point functions formally required in Theorem 2 is actually redundant, since

$$
A\left(\varphi_{j} ; t\right) \Omega=\frac{d}{d t} A^{*}\left(\varphi_{j} ; t\right) \Omega=0
$$

by (3.1). So the strong limit

$$
\mathrm{s}-\lim _{t \rightarrow \pm \infty} \prod_{j=1}^{n} A^{*}\left(\varphi_{j} ; t\right) \Omega
$$


exists in $\mathscr{H}$ and we are left to justify its physical interpretation. Here we remark that the well-known properties of asymptotic states are fulfilled, as there are correct transformation under $U(\Lambda, a)$ and scalar products among each other. These properties can be easily established from (3.1) and inequalities of type

$$
\begin{aligned}
|t|^{-3 / 2} & \left\langle\Omega\left|\prod_{j=1}^{l-1} A^{\#}\left(\varphi_{j} ; t\right)\left[A^{\#}\left(\varphi_{l} ; t\right), A^{\#}\left(\varphi_{l+1} ; t\right)\right]_{-} \prod_{j^{\prime}=l+2}^{n^{\prime}} A^{\#}\left(\varphi_{j} ; t\right)\right| \Omega\right\rangle \\
- & \left\langle\Omega\left|\prod_{j=1}^{l-1} A^{\#}\left(\varphi_{j} ; t\right) \prod_{j^{\prime}=l+2}^{n^{\prime}} A^{\#}\left(\varphi_{j} ; t\right)\right| \Omega\right\rangle \\
\cdot & \left\langle\Omega\left|\left[A^{\#}\left(\varphi_{l} ; t\right), A^{\#}\left(\varphi_{l+1} ; t\right)\right]_{-}\right| \Omega\right\rangle \mid<C
\end{aligned}
$$

which are a direct consequence of Theorem 2 since the required subtractions cancel each other. For further discussion of the physical interpretation we refer to Haag's papers $[2,6,7]$.

Denote by $\mathscr{H}_{\text {out }}\left(\right.$ resp. $\left.\mathscr{H}_{\text {in }}\right)$ the closed subspace of $\mathscr{H}$ generated by all out(resp. in)-states obtained from Haag's strong asymptotic condition. Moreover, given an arbitrary family $\left\{\Phi_{t}\right\}_{t \in R^{1}}$ of vectors in $\mathscr{H}$, write for $\Phi_{+} \in \mathscr{H}_{\text {out }}\left(\right.$ resp. $\left.\Phi_{-} \in \mathscr{H}_{\text {in }}\right)$

$$
\Phi_{+}=\mathrm{w}_{+}-\lim _{t \rightarrow+\infty} \Phi_{t} \quad\left(\operatorname{resp} . \Phi_{-}=\mathrm{w}_{-}-\lim _{t \rightarrow-\infty} \Phi_{t}\right)
$$

whenever

$$
\lim _{t \rightarrow \pm \infty}\left\langle\Psi_{ \pm} \mid \Phi_{t}\right\rangle=\left\langle\Psi_{ \pm} \mid \Phi_{ \pm}\right\rangle
$$

holds for all $\Psi_{+} \in \mathscr{H}_{\text {out }}$ (resp. $\Psi_{-} \in \mathscr{H}_{\text {in }}$ ). Using this convention we next derive the corresponding form of

Haag's Weak Asymptotic Condition. Let $\varphi_{1}, \ldots, \varphi_{n}$ be test functions from $S^{s}\left(R^{4}\right)$. Then

$$
\mathrm{w}_{ \pm}-\lim _{t \rightarrow \pm \infty} \prod_{j=1}^{n} A^{*}\left(\varphi_{j} ; t\right) \Omega
$$

exists and represents an asymptotic state corresponding to $n$ particles with momentum space wave functions $\hat{f}_{j}(\boldsymbol{p}) \equiv \tilde{\varphi}_{j}\left(\omega_{\boldsymbol{p}}, \boldsymbol{p}\right)$.

Proof. First of all, by Theorem 2 again, we see

$$
\left\|\prod_{j=1}^{n} A^{*}\left(\varphi_{j} ; t\right) \Omega\right\|^{2}
$$

to have an upper bound independent of $t$. By Haag's strong asymptotic condition, therefore, if we choose test functions $\varphi_{1}^{\prime}, \ldots, \varphi_{n}^{\prime} \in S^{s}\left(R^{4}\right)$ with $\operatorname{supp} \tilde{\varphi}_{j}^{\prime} \subset\left\{p \in R^{4}: p^{2}<M^{2}\right\}$ and $\tilde{\varphi}_{j}^{\prime}\left(\omega_{\boldsymbol{p}}, \boldsymbol{p}\right)=\tilde{\varphi}_{j}\left(\omega_{\boldsymbol{p}}, \boldsymbol{p}\right)$, it is quite sufficient 
to prove the identity

$$
\lim _{t \rightarrow \pm \infty}\left\langle\Psi_{ \pm}\left|\prod_{j=1}^{n} A^{*}\left(\varphi_{j} ; t\right)\right| \Omega\right\rangle=\lim _{t \rightarrow \pm \infty}\left\langle\Psi_{ \pm}\left|\prod_{j=1}^{n} A^{*}\left(\varphi_{j}^{\prime} ; t\right)\right| \Omega\right\rangle
$$

for arbitrary $\Psi_{ \pm}$of the form

$$
\Psi_{ \pm}=\mathrm{s}-\lim _{t \rightarrow \pm \infty} \prod_{j=1}^{n^{\prime}} A^{*}\left(\psi_{j} ; t\right) \Omega
$$

with $\psi_{j} \in S^{s}\left(R^{4}\right)$, supp $\tilde{\psi}_{j} \subset\left\{p \in R^{4}: p^{2}<M^{2}\right\}$. This identity follows from

$$
\begin{aligned}
& \lim _{t \rightarrow \pm \infty} \mid\left\langle\Omega\left|\prod_{r=1}^{n^{\prime}} A\left(\psi_{r} ; t\right) \prod_{j=1}^{n} A^{*}\left(\varphi_{j} ; t\right)\right| \Omega\right\rangle \\
& -\left\langle\Omega\left|\prod_{r=1}^{n^{\prime}} A\left(\psi_{r} ; t\right) \prod_{j=1}^{n} A^{*}\left(\varphi_{j}^{\prime} ; t\right)\right| \Omega\right\rangle \mid=0,
\end{aligned}
$$

which, in turn, is a consequence of Theorem 2 since the required subtractions cancel each other by (3.1).

Just as for tempered fields we still have the following

Lemma 8. Let $\varphi_{1}, \ldots, \varphi_{n}$ be test functions from $S^{s}\left(R^{4}\right)$. Then there are $C, N \in Z_{+}$such that

$$
\left|\left\langle\Omega\left|\prod_{j=1}^{n} A^{\#}\left(\varphi_{j} ; \chi^{j}\right)\right| \Omega\right\rangle\right|<C(1+\|\chi\|)^{N}
$$

holds for all $\chi \in R^{n}$.

Proof. Note that

$$
\begin{gathered}
\left\langle\Omega\left|\prod_{j=1}^{n} A^{\#}\left(\varphi_{j} ; \chi^{j}\right)\right| \Omega\right\rangle \\
=\int d \hat{p}\left\langle\Omega\left|\tilde{A}\left(p_{1}\right) \ldots \tilde{A}\left(p_{n}\right)\right| \Omega\right\rangle \prod_{j=1}^{n} \tilde{\varphi}_{j}^{\#}\left(p_{j} ; \chi^{j}\right) \\
\tilde{\varphi}_{j}^{\#}(p ; t) \in\left\{\tilde{\varphi}_{j}(-p ; t), \tilde{\varphi}_{j}^{*}(p ; t)\right\},
\end{gathered}
$$

with$$
\tilde{\varphi}_{j}(p ; t) \equiv(2 \pi)^{-1 / 2} \tilde{\varphi}_{j}(p)\left(\frac{p^{0}+\omega_{\boldsymbol{p}}}{2 \omega_{\boldsymbol{p}}}\right) e^{i\left(p^{0}-\omega_{\boldsymbol{p}}\right) t} .
$$

Since $\left\langle\Omega\left|\tilde{A}\left(\mathrm{p}_{1}\right) \ldots \tilde{A}\left(p_{n}\right)\right| \Omega\right\rangle$ is a generalized function on $\widetilde{S^{s}}\left(R^{4 n}\right)$, for every $A>0$ there are integers $N_{A}, C_{A}$ such that (see [4])

$$
\begin{gathered}
\left|\left\langle\Omega\left|\prod_{j=1}^{n} A^{\#}\left(\varphi_{j} ; \chi^{j}\right)\right| \Omega\right\rangle\right| \\
\leqq C_{A} \max _{\hat{p} \in R^{4 n}} \max _{|\hat{\alpha}| \leqq N_{A}} \max _{\hat{\hat{\beta}} \in Z_{+}^{4 n}} \hat{\beta}^{-s \hat{\beta}}\left|(\hat{p} / A)^{\hat{\beta}} D_{\hat{\hat{p}}}^{\hat{\alpha}} \prod_{j=1}^{n} \tilde{\varphi}_{j}^{\#}\left(p_{j} ; \chi^{j}\right)\right| .
\end{gathered}
$$


Choosing $A$ big enough, we see the r.h.s. to be bounded by a polynomial in the $\left|\chi^{j}\right|$ of degree $\leqq N_{A}$, due to the restriction $|\hat{\alpha}| \leqq N_{A}$.

This Lemma enables us to take over Hepp's proof for the following form of the LSZ asymptotic condition [10]:

Theorem 3. Let $\varphi_{1}, \ldots, \varphi_{n}$ be test functions from $S^{s}\left(R^{4}\right)$. Then, if $\left\{\tilde{\varphi}_{2}\left(\omega_{\boldsymbol{p}}, \boldsymbol{p}\right), \ldots, \tilde{\varphi}_{n}\left(\omega_{\boldsymbol{p}}, \boldsymbol{p}\right)\right\} \subset \mathscr{S}\left(R^{3}\right)$ is essentially non-overlapping, we have

$$
\mathrm{w}_{ \pm}-\lim _{t \rightarrow \pm \infty} A^{*}\left(\varphi_{1} ; t\right) \mathrm{w}_{t_{t^{\prime} \rightarrow \pm \infty}}-\lim _{j=2} \prod^{n} A^{*}\left(\varphi_{j} ; t^{\prime}\right) \Omega=\mathrm{w}_{ \pm}-\lim _{t \rightarrow \pm \infty} \prod_{j=1}^{n} A^{*}\left(\varphi_{j} ; t\right) \Omega .
$$

If, moreover, supp $\tilde{\varphi}_{j} \subset\left\{p \in R^{4}: p^{2}<M^{2}\right\}$ for $j=1, \ldots, n$ then we may substitute all the $\mathrm{w}_{ \pm}$-limits by strong limits.

Remark. The physical interpretation of this theorem is obviously given by Haag's weak asymptotic condition.

Proof. By Lemma 5 there is a suitable function $\hat{g} \in \widetilde{S}^{s}\left(R^{3}\right)$ such that $\hat{f}_{j}^{\prime}(\boldsymbol{p}) \equiv \tilde{\varphi}_{j}\left(\omega_{\boldsymbol{p}}, \boldsymbol{p}\right) / \hat{g}(\boldsymbol{p}) \in{\widetilde{S^{s}}}^{(}\left(R^{3}\right)$ for $j=2, \ldots, n$. Let us choose some $h \in \mathscr{D}\left(R^{1}\right)$ with supp $h \subset\left(0, M^{2}\right), h\left(m^{2}\right)=1$ and define

$$
\tilde{\psi}(p) \equiv \hat{g}(\boldsymbol{p}) h\left(p^{2}\right), \quad \widetilde{\varphi_{j}^{\prime}}(p) \equiv \hat{f_{j}^{\prime}}(\boldsymbol{p}) \tilde{\psi}(p)
$$

for $j=2, \ldots, n$. Then we have $\psi, \varphi_{j}^{\prime} \in S^{s}\left(R^{4}\right)$, and $\left\{f_{2}^{\prime}, \ldots, f_{n}^{\prime}\right\}$ is essentially non-overlapping. Therefore, by Theorem 2 (second part) we have

$$
|t|^{N}\left\|\frac{d}{d t} \prod_{j=2}^{n} A^{*}\left(\varphi_{j}^{\prime} ; t\right) \Omega\right\|^{2}<C_{N}
$$

for every $N \in Z_{+}$(compare proof of Haag's strong asymptotic condition). Furthermore, by Lemma 8, we obtain the following estimate

$$
\begin{aligned}
\| A^{*}\left(\varphi_{1} ; t\right) & \left(\prod_{j=2}^{n} A^{*}\left(\varphi_{j}^{\prime} ; t_{1}\right)-\prod_{j=2}^{n} A^{*}\left(\varphi_{j}^{\prime} ; t_{2}\right)\right) \Omega \| \\
= & \sup _{\substack{\Psi \in D \\
\|\Psi\|=1}}\left|\left\langle\Psi\left|A^{*}\left(\varphi_{1} ; t\right)\left(\prod_{j=2}^{n} A^{*}\left(\varphi_{j}^{\prime} ; t_{1}\right)-\prod_{j=2}^{n} A^{*}\left(\varphi_{j}^{\prime} ; t_{2}\right)\right)\right| \Omega\right\rangle\right| \\
= & \sup _{\substack{\Psi \in D \\
\|\Psi\|=1}} \int_{t_{1}}^{t_{2}} d t^{\prime}\left\langle\Psi\left|A^{*}\left(\varphi_{1} ; t\right) \frac{d}{d t^{\prime}} \prod_{j=2}^{n} A^{*}\left(\varphi_{j}^{\prime} ; t^{\prime}\right)\right| \Omega\right\rangle \mid \\
\leqq & \left|\int_{t_{1}}^{t_{2}} d t^{\prime}\right| A^{*}\left(\varphi_{1} ; t\right) \frac{d}{d t^{\prime}} \prod_{j=2}^{n} A^{*}\left(\varphi_{j}^{\prime} ; t^{\prime}\right) \Omega \| \mid \\
\leqq & \mid \int_{t_{1}}^{t_{2}} d t^{\prime}\left(\|\|_{1} A\left(\varphi_{1} ; t\right) A^{*}\left(\varphi_{1} ; t\right) \frac{d}{d t^{\prime}} \prod_{j=2}^{n} A^{*}\left(\varphi_{j}^{\prime} ; t^{\prime}\right) \Omega \|\right. \\
& \left.\cdot\left\|\frac{d}{d t^{\prime}} \prod_{j=2}^{n} A^{*}\left(\varphi_{j}^{\prime} ; t^{\prime}\right) \Omega\right\|\right)^{1 / 2} \mid \\
\leqq & C(1+|t|)^{N}\left(1+\left|t_{1}\right|\right)^{-(N+1)},
\end{aligned}
$$


which, with suitable $C, N \in Z_{+}$, is valid for all $t, t_{1}, t_{2} \in R^{1}$ with $0<t_{1}<t_{2}$ or $t_{2}<t_{1}<0$. Since $A^{*}\left(\varphi_{1} ; t\right)$ is closed we see from this estimate that

$$
\operatorname{sil}_{t^{\prime} \rightarrow \pm \infty} \prod_{j=2}^{n} A^{*}\left(\varphi_{j}^{\prime} ; t^{\prime}\right) \Omega
$$

if it exists, is in the domain of $A^{*}\left(\varphi_{1} ; t\right)$. Therefore, by Haag's asymptotic conditions and since $\widetilde{\varphi_{j}^{\prime}}\left(\omega_{\boldsymbol{p}}, \boldsymbol{p}\right)=\tilde{\varphi}_{j}\left(\omega_{\boldsymbol{p}}, \boldsymbol{p}\right)$ for $j=2, \ldots, n$, we see that

$$
\begin{aligned}
& \left\|A^{*}\left(\varphi_{1} ; t\right)\left(\mathrm{w}_{ \pm}-\lim _{t^{\prime} \rightarrow \pm \infty} \prod_{j=2}^{n} A^{*}\left(\varphi_{j} ; t^{\prime}\right) \Omega-\prod_{j=2}^{n} A^{*}\left(\varphi_{j}^{\prime} ; t\right) \Omega\right)\right\| \\
& =\left\|A^{*}\left(\varphi_{1} ; t\right)\left(\operatorname{s}_{t^{\prime} \rightarrow \pm \infty} \prod_{j=2}^{n} A^{*}\left(\varphi_{j}^{\prime} ; t^{\prime}\right) \Omega-\prod_{j=2}^{n} A^{*}\left(\varphi_{j}^{\prime} ; t\right) \Omega\right)\right\| \\
& \quad \rightarrow 0 \text { for } t \rightarrow \pm \infty,
\end{aligned}
$$

i.e. the $\mathrm{w}_{ \pm}$(resp. strong)-limit of

$$
A^{*}\left(\varphi_{1} ; t\right) \mathrm{w}_{ \pm_{t^{\prime} \rightarrow \pm \infty}}-\lim _{j=2} A^{*}\left(\varphi_{j} ; t^{\prime}\right) \Omega
$$

for $t \rightarrow \pm \infty$ exists and is equal to the $\mathrm{w}_{ \pm}$(resp. strong)-limit of

$$
\Phi^{\prime}(t) \equiv A^{*}\left(\varphi_{1} ; t\right) \prod_{j=2}^{n} A^{*}\left(\varphi_{j}^{\prime} ; t\right) \Omega
$$

for $t \rightarrow \pm \infty$, iff the latter exists. By Haag's weak asymptotic condition the $\mathrm{w}_{ \pm}$-limit of $\Phi^{\prime}(t)$ exists and, due to $\widetilde{\varphi_{j}^{\prime}}\left(\omega_{\boldsymbol{p}}, \boldsymbol{p}\right)=\tilde{\varphi}_{j}\left(\omega_{\boldsymbol{p}}, \boldsymbol{p}\right)$ for $j=2, \ldots, n$, is equal to

$$
\mathrm{w}_{ \pm}-\lim _{t \rightarrow \pm \infty} \prod_{j=1}^{n} A^{*}\left(\varphi_{j} ; t\right) \Omega .
$$

Hence, the first statement of Theorem 3 is proved. The second follows analogously from the fact that for $\operatorname{supp} \widetilde{\varphi_{j}} \subset\left\{p \in R^{4}: p^{2}<M^{2}\right\}, j=1, \ldots, n$, Haag's asymptotic conditions imply

$$
\begin{gathered}
\operatorname{s-lim}_{t \rightarrow \pm \infty} \Phi^{\prime}(t)=\underset{t \rightarrow \pm \infty}{\mathrm{s}-\lim _{t \rightarrow \infty}} \prod_{j=1}^{n} A^{*}\left(\varphi_{j} ; t\right) \Omega \\
\mathrm{w}_{ \pm}-\lim _{t \rightarrow \pm \infty} \prod_{j=2}^{n} A^{*}\left(\varphi_{j} ; t\right) \Omega=\mathrm{s}-\lim _{t \rightarrow \pm \infty} \prod_{j=2}^{n} A^{*}\left(\varphi_{j} ; t\right) \Omega .
\end{gathered}
$$

\section{Conclusions}

We introduced the axiom of essential locality as a natural generalization of local commutativity. Contrary to the latter, essential locality - while equivalent to locality in the localizable case - is meaningful also 
for the non-localizable case. Thus, by essential locality, we rigorously derived the usual Haag-Ruelle-Hepp scattering formalism with all its different forms of the asymptotic condition in a self-contained way.

We did not derive the LSZ reduction formulae, since we were not willing to postulate existence of the commonly used Green's functions. However, modified ${ }^{9}$ reduction formulae can be derived in the usual way [10].

There are examples of essentially local non-localizable fields which are not just restrictions of localizable fields to non-localizable test spaces. Such examples are provided by functions of the free field ${ }^{10}$ as treated by Rieckers [15].

We conclude that the idea of Einstein-causality still has some applicability to the non-localizable case and ensures a particle interpretation of the theory.

Finally, as a by-product, we have an explicit derivation of asymptotic conditions for Jaffe fields [11]. For example, we have all the usual asymptotic conditions for the simple case of asymptotic particles corresponding to wave functions with compact support in momentum space. This is because every Jaffe field yields, just by restriction, an essentially local field on $S^{1}\left(R^{4}\right)$ and because $\mathscr{D}\left(R^{n}\right) \subset{\widetilde{S^{1}}}^{1}\left(R^{n}\right)$.

\section{Appendix: Proof of Lemma 2}

We prove Lemma 2 for the special case $l=1$ only, the full statement following directly by induction, then ${ }^{11}$.

Let us first affirm that:

(i) the linear hull $S^{S}\left(R^{n}\right) \otimes S^{S}\left(R^{n_{1}}\right)$ of $S^{S}\left(R^{n}\right) \times S^{S}\left(R^{n_{1}}\right)$ is dense in $S^{S}\left(R^{n+n_{1}}\right)$

$\mathscr{D}\left(R^{n+n_{1}}\right)$ is dense in $\widetilde{S^{s}}\left(R^{n+n_{1}}\right)$ and the topology of $\mathscr{D}\left(R^{n+n_{1}}\right)$ is finer than the topology induced by $\widetilde{S^{S}}\left(R^{n+n_{1}}\right)$. Therefore, since $\mathscr{D}\left(R^{n}\right) \otimes \mathscr{D}\left(R^{n_{1}}\right)$ is well known to be dense in $\mathscr{D}\left(R^{n+n_{1}}\right)$, we see $\widetilde{S^{s}}\left(R^{n}\right) \otimes \widetilde{S^{s}}\left(R^{n_{1}}\right)$ to be dense in $\widetilde{S^{s}}\left(R^{n+n_{1}}\right)$. Thus, by Fourier transform, we get (i).

Note that, by this statement, in the localizable case $s>1$ any generalized function $F$ on $S^{s}\left(R^{n+n_{1}}\right)$ has its support in $M \times R^{n_{1}}$ if its restriction to $S^{s}\left(R^{n}\right) \times S^{s}\left(R^{n_{1}}\right)$ has so. Therefore, by Lemma 1, it is sufficient to prove

${ }^{9}$ In the formal definition of the Green's functions the step function has to be substituted by some suitable multiplier.

10 The non-obvious proof will be given elsewhere.

${ }^{11}$ This is because the dual space of $S^{S}\left(R^{n^{\prime}}\right)$ is complete with respect to weak limits $[4,15]$. 
Lemma 2 for the following two cases:

1. Case: $M=R^{n}, \quad s$ arbitrary,

2. Case: $M$ arbitrary, $s \leqq 1$.

Now, if for $A, e>0$ we denote by $S_{M, e}^{s, A}\left(R^{n}\right)$ the set of all functions $\varphi \in S^{s}\left(R^{n}\right)$ fulfilling

$$
\begin{aligned}
\|\varphi\|_{A^{\prime}, N} & \equiv \sup _{\chi \in U_{e}(M)} \sup _{\alpha \in Z_{+}^{\eta}} A^{\prime-|\alpha|} \alpha^{-s \alpha}(1+\|\chi\|)^{N}\left|\varphi^{(\alpha)}(\chi)\right| \\
& <\infty
\end{aligned}
$$

for arbitrary $N \in Z_{+}, A^{\prime}>A$ and choose the topology given by the set of semi-norms (A.1), we see $S_{M, e}^{s, A}\left(R^{n}\right)$ to be metrizable (since Hausdorff) in both cases under consideration. On the other hand, since $\widetilde{S^{S, A}}\left(R^{n_{1}}\right)$ $\equiv \widetilde{S_{R^{n_{1}}, 1}^{s, A}}\left(R^{n_{1}}\right)$ and hence also $S^{s, A}\left(R^{n_{1}}\right)$ is nuclear $[5, \mathrm{p} .84]$, we have $[14$, p. 101]:

$$
S_{M, e}^{s, A}\left(R^{n}\right) \otimes_{\pi} S^{s, A}\left(R^{n_{1}}\right)=S_{M, e}^{s, A}\left(R^{n}\right) \otimes_{\varepsilon} S^{s, A}\left(R^{n_{1}}\right) .
$$

Recall that the $\pi$-tensor product $[14$, p. 97$]$ is the algebraic tensor product endowed with the finest topology such that the canonical embedding

$$
S_{M, e}^{s, A}\left(R^{n}\right) \times S^{s, A}\left(R^{n_{1}}\right) \rightarrow S_{M, e}^{s, A}\left(R^{n}\right) \otimes_{\pi} S^{s, A}\left(R^{n_{1}}\right)
$$

is continuous, thus every continuous functional on $S_{M, e}^{s, A}\left(R^{n}\right) \times S^{s, A}\left(R^{n_{1}}\right)$ has a unique continuous extension to $S_{M, e}^{s, A}\left(R^{n}\right) \otimes_{\pi} S^{s, A}\left(R^{n_{1}}\right)[16$, p. 131]. Moreover, since $S^{s, A}\left(R^{n_{1}}\right)$ is a Fréchet space (and remember that $S_{M, e}^{s, A}\left(R^{n}\right)$ is metrizable), the functional in question is only required to be separately continuous in both arguments $[16, \mathrm{p} .136] . F_{0}$ clearly meets the latter requirement, so, by (A.2), we are left to prove the following statements:

(ii) $S^{s, A}\left(R^{n}\right) \otimes S^{s, A}\left(R^{n_{1}}\right)$ is dense in $S^{s}\left(R^{n+n_{1}}\right)$; hence $S_{M, e}^{s, A}\left(R^{n}\right) \otimes S^{s, A}\left(R^{n_{1}}\right)$ is dense in $S_{M \times R^{n_{1}}, e}^{S, A}\left(R^{n+n_{1}}\right)$.

(iii) The topology of $S_{M, e}^{s, A}\left(R^{n}\right) \otimes_{\varepsilon} S^{s, A}\left(R^{n_{1}}\right)$ is the same as the topology induced by $S_{M \times R^{n_{1}, e}}^{S, A}\left(R^{n+n_{1}}\right)$.

Although statement (ii) is a generalization of statement (i) it follows from the same argument (because $\mathscr{D} \subset \widetilde{S^{s, A}}$ ). The proof of statement (iii) is slightly more involved:

Consider the set

$$
U \equiv\left\{\varphi \in S_{M, e}^{s, A}\left(R^{n}\right):\|\varphi\|_{A^{\prime}, N} \leqq 1\right\} .
$$

Since $U$ is closed and absolutely convex, we have $U=U^{00}[16$, p. 36]. Here, as usual, by $U^{0}$ and $U^{00}$ we denote the polar and bipolar of $U$ respectively. Hence $U$ may also be characterized by

$$
U=\left\{\varphi \in S_{M, e}^{s, A}\left(R^{n}\right):|F(\varphi)| \leqq 1 \text { for all } F \in U^{0}\right\} .
$$


On the other hand, the functional $F_{\chi, \alpha}$, defined by

$$
F_{\chi, \alpha}(\varphi) \equiv A^{\prime-|\alpha|} \alpha^{-s \alpha}(1+\|\chi\|)^{N} \varphi^{(\alpha)}(\chi),
$$

is clearly in $U^{0}$ and we even have

$$
U=\left\{\varphi \in S_{M, e}^{s, A}\left(R^{n}\right):\left|F_{\chi, \alpha}(\varphi)\right| \leqq 1 \text { for all } \chi \in U_{e}(M), \alpha \in Z_{+}^{n}\right\} .
$$

Using some obvious generalization of our notation and defining

$$
\begin{aligned}
W_{A^{\prime}, N, \delta} \equiv & \left\{\chi \in S_{M, e}^{s, A}\left(R^{n}\right) \otimes S^{s, A}\left(R^{n_{1}}\right):\left|F_{\chi, \alpha} \otimes F_{\chi_{1}, \alpha_{1}}(\chi)\right| \leqq \delta\right. \\
& \text { for all } \left.\chi \in U_{e}(M), \alpha \in Z_{+}^{n}, \chi_{1} \in R^{n_{1}}, \alpha_{1} \in Z_{+}^{n_{1}}\right\}
\end{aligned}
$$

we conclude:

$$
\begin{aligned}
W_{A^{\prime}, N, \delta}= & \left\{\chi \in S_{M, e}^{s, A}\left(R^{n}\right) \otimes S^{s, A}\left(R^{n_{1}}\right):\left|F \otimes F_{1}(\chi)\right| \leqq \delta\right. \\
& \text { for all } \left.F \in U^{0}, F_{1} \in U_{1}^{0}\right\} .
\end{aligned}
$$

While the definition of the $W_{A^{\prime}, N, \delta}\left(A^{\prime}>A, \delta>0, N \in Z_{+}\right)$shows that they form a base of 0-neighbourhoods in $S_{M, e}^{s, A}\left(R^{n}\right) \otimes S^{s, A}\left(R^{n_{1}}\right)$ with respect to the topology induced by $S_{M \times R^{n_{1}, e}}^{s, A}\left(R^{n+n_{1}}\right)$, Eq. (A.3) shows that this also holds with respect to the topology of the $\varepsilon$-tensor product $[14$, p. 97$]$. This proves statement (iii).

\section{References}

1. Araki,H.: Zürich Lectures, 1962 (unpublished)

2. Brenig, W., Haag, R.: Fortschr. Physik 7, 183-242 (1959)

3. Gelfand,I.M., Schilow, G.E.: Verallgemeinerte Funktionen, Vol. I. Berlin: VEB Deutsch. Verl. d. Wissensch. 1967

4. Gelfand,I.M., Schilow, G.E.: Verallgemeinerte Funktionen, Vol. II. Berlin: VEB Deutsch. Verl. d. Wissensch. 1962

5. Gelfand,I.M., Wilenkin, N.J.: Verallgemeinerte Funktionen, Vol. IV. Berlin: VEB Deutsch. Verl. d. Wissensch. 1964

6. Haag, R.: Phys. Rev. 112, 669-673 (1958)

7. Haag, R.: Suppl. Nuovo Cim. 14, 131-152 (1959)

8. Haag, R., Schroer, B.: J. Math. Phys. 3, 248-255 (1962)

9. Hepp, K.: Helv. Phys. Acta 37, 639-658 (1964)

10. Hepp, K.: Commun. math. Phys. 1, 95-111(1965)

11. Jaffe, A.: Phys. Rev. 158, 1454-1461 (1967)

12. Jost, R.: The General Theory of Quantized Ficlds. Providence: Am. Math. Soc. 1965

13. Lücke, W.: Int. J. Theor. Phys. (in press)

14. Pietsch, A.: Nukleare lokalkonvexe Räume. Berlin: Akademie-Verlag 1969

15. Rieckers, A.: Int. J. Theor. Phys. 4, 55-82 (1971)

16. Robertson, A.P., Robertson, W. J.: Topological Vector Spaces. Cambridge: Cambridge University Press 1964

17. Steinmann, O.: Helv. Phys. Acta 36, 90-112 (1963)

18. Steinmann, O.: Commun. math. Phys. 10, 245-268 (1968)

19. Steinmann, O.: Commun. math. Phys. 18, 179 (1970) 
20. Streater,R.F., Wightman,A.S.: PCT spin and statistics and all that. New York: W. A. Benjamin 1964

21. Ruelle, D.: Helv. Phys. Acta 35, 147-163 (1962)

22. Taylor, J. G.: Ann. Phys. 68, 484-498 (1971)

23. Taylor,J. G., Constantinescu, F.: Commun. math. Phys. 30, $211-227$ (1973)

Communicated by R. Haag

\author{
W. Lücke \\ Institut für Theoretische Physik \\ der Technischen Universität \\ D-3392 Clausthal-Zellerfeld, Leibnizstraße \\ Federal Republic of Germany
}

Note Added in Proof. In a paper we did not know before publication in J. Math. Phys. 15, 824 - 830 (1974), Constantinescu and Taylor introduced the notion of order of extension of the commutator bracket outside the light cone. For $s<1$ this order may be proved to be $\geqq(1-s)^{-1}$ iff the field on $S^{s}\left(R^{4}\right)$ is essentially local. 